\title{
DENGUE FEVER, EXPANDED DENGUE SYNDROME AND DENGUE SHOCK SYNDROME: CLINICAL PROFILE, MANAGEMENT AND OUTCOME OF PATIENTS AT A TERTIARY HOSPITAL, DHAKA, BANGLADESH
}

\author{
ROZANA ROUF ${ }^{1}$, RAIHAN RABBANI ${ }^{2}$, PRATIK DEWAN ${ }^{3}$, MIRZA NAZIM UDDIN $^{4}$, JAHANGIR ALAM ${ }^{5}$, KAZI ALI \\ HASSAN ${ }^{6}$, MD. ABU BAKAR 7 , MD. ROBED AMIN ${ }^{8}$, ANOWAR HOSSAIN ${ }^{9}$
}

\begin{abstract}
:
Background: Dengue fever is endemic in Bangladesh. Its incidence has been increasing and spreading from urban Dhaka to rural areas. In 2019, over 100,000 cases reported with deaths over 250. Clinical profiles varied from classical signs and symptoms to alarming severity and complications, such as, Expanded Dengue Syndrome (EDS), Dengue Shock Syndrome (DSS) along with coinfections and comorbidities. No detail study on EDS \& DSS carried out yet over 2019 outbreak. This report highlights the features, management and the outcome of EDS \& DSS cases of 2018-19 admitted in a specialized tertiary private hospital in Dhaka, Bangladesh.
\end{abstract}

Methods: This cross sectional observational study was performed at Square Hospital, Dhaka, exclusively over 62 adult dengue patients with complications who fulfilled the admission criteria in ICU and HDU and managed as per national guidelines and diagnosed by positive NS1 Ag, Dengue RT PCR , Anti-dengue IgM, hematological and biochemical tests.

Results: Of 343 cases, 62 (18.1\%) patients had complications; 17 (27.4\%) admitted in ICU and 45 (72.6\%) patients in HDU. Among 62 patients, 25 patients had EDS (40.3\%) and 17 had DSS (27.4\%). Of the EDS patients, majority were 40-50 years old with male predominance. Of 62 patients, 42 had atypical presentations (67.74\%), 25 (40.3\%) had EDS and 17 (27.4\%) had DSS, 16 (25.8\%) had coinfection, 45 (72.58\%) had co-morbidities. Most patients presented with gastrointestinal symptoms (80\%). Twenty one cases (84\%) of EDS survive; 4 (16\%), died after developing rhabdomyolysis, multiorgan dysfunction and acute renal failure.

Conclusion: Early diagnosis of complicated cases by clinical features, lab predictors and early fluid therapy are the key to successful management with positive outcome. Death could be averted by recognizing the stage of plasma leakage indicated by raised CRP, reduced serum Albumin, and ultrasonography for detecting ascites, pleural effusion, mucosal thickening and edema of gall bladder and appropriate replacement of fluid loss and maintenance of nutrition.

Keywords: Dengue fever, Dengue expanded syndrome, Dengue shock syndrome.

Received: 26 March 2020

Accepted: 12 June 2020

DOI: https://doi.org/10.3329/bjm.v31i2.48533

1. MBBS, FCPS (Internal Medicine),MACP, MACE, Associate Consultant, Dept. of medicine, Square Hospital

2. MBBS, FCPS, MD, Consultant, Internal Medicine and ICU.

3. MBBS, DEM, MD, Consultant, Internal Medicine and Endocrinology.

4. MBBS, MRCP (UK), Director, Medical Services, Consultant, Internal Medicine and ICU.

5. MBBS, MRCP(UK),FRCP(Edin). Consultant, Internal Medicine and Diabetology.

6. MBBS, M.Phil. (EM), MRCP (UK).Consultant, Endocrinology and Internal Medicine.

7. MBBS, FCPS, FACP(USA), FRCP (Edin), Consultant, Internal Medicine.

8. MBBS, FCPS, Professor, Dept of Medicine, Dhaka Medical College and Hospital, Dhaka.

9. MBBS, MCPS, MCAP, FCAP, Head, Laboratory Operation, Square Hospitals Ltd., Dhaka.

Corresponding author: Dr. Rozana Rouf, Dept. of Medicine, Square Hospital Ltd. Dhaka. 18/F West Panthapath, Dhaka 1205. Email:rozanarouf@gmail.com. 


\section{Introduction:}

The increasing burden of dengue is a matter of serious public health concern in the world, especially in the absence of specific antiviral drug and a great challenge for the clinicians to recognize the severity of the disease at the early phase for timely, effective management to reduce complication and death ${ }^{1}$. Dengue has become endemic in more than 100 countries throughout the globe $^{2}$. An estimated 50-100 million dengue infections occur annually and approximately 2.5 billion people live in dengue endemic countries. Among them, 250,000 individuals per year manifest severe form of dengue with about 10 percent mortality ${ }^{2,3}$. Dengue haemorrhagic fever (DHF) first emerged as a public health problem in 1954, during first epidemic in Manila ${ }^{4}$. In the last 50 years, incidence increased 30 folds with geographic expansion to new countries and, in the present decade, dengue spreading from urban to rural setting. It is thus the most rapidly spreading mosquito-borne viral disease in the world.

Dengue fever was unknown in Bangladesh until an outbreak in 1964 known as Dhaka fever. The dengue outbreak in 2000, first time in Bangladesh, started in late June, peaked in September and subsided in the dry winter season in November-December. About 5,575 hospitalized dengue cases were reported in that outbreak with 93 deaths (fatality 1.6\%). A good number of cases occurred from 2001-2006 with a few deaths. Between the years 2007-2011, a lower number of cases reported but no death recorded. From 2015, the incidence of dengue cases started rising with 6,060 cases in 2016 with 14 deaths and 10,148 cases in 2018 with 26 deaths due to severity and complications. The risk of severe and complications of dengue is much higher in secondary cases rather than primary infection ${ }^{5,6}$. To describe cases with severe atypical manifestations, WHO in 2012 used the term Expanded Dengue Syndrome (EDS) in its guidelines ${ }^{2,6}$. In 2019 outbreaks in Bangladesh, there were over 100,000 dengue cases with deaths over 200 including deaths in rural areas, which may be due to complications or inappropriate management. The cause of death may also be attributed to severity of pathogenesis complex associated with the immune factor known as ADE (Antibody Dependant Enhancement) as well as the changing epidemiology in human host, the dengue virus and the vector bionomics ${ }^{6,7}$.

Patients of dengue hemorrhagic fever showed unusual manifestations with involvement of several organs dysfunction such as liver, kidneys, brain or heart etc are increasingly reported. The unusual manifestation is also noted in some dengue patients without evidence of plasma leakage who have had associated co- infections, co-morbidities or complications of prolonged shock and those are clubbed under the EDS 5,6 .

Information on severe and complicated cases of dengue in Bangladesh is scanty, it is thus worthwhile to study the characteristics of these cases in terms of clinical manifestations, laboratory biomarkers, management and outcome of admitted patients that might be helpful for caregivers. This study attempted to document the presenting features and outcome of complicated cases of dengue who were admitted into ICU and HDU of the Square hospital, a centrally located, tertiary private hospital, Dhaka, Bangladesh.

\section{Materials and methods:}

Study place and period: This cross sectional observational study carried out in the inpatient department of the specialized hospital between July, 2018 and April, 2019. The information was captured using an case record furm (CRF), developed based on literature review and in consultation with selected experts. Patients who fulfilled the admission criteria were enrolled in the study ${ }^{3}$.

Study population: Admitted patients were carefully observed, pertinent clinical and laboratory data recorded daily on a standard case record form. Clinical examination carried out meticulously including vital signs, petechiae or other skin hemorrhages, signs of circulatory failure, pleural effusion, ascites, hepatomegaly and signs of pancreatitis etc. Patients were selected based on the laboratory confirmation of NS1 Ag or Dengue RT PCR or Anti-dengue IgM and other hematological and biochemical tests. Patients with dengue positive cases with evidences of plasma leakage were specifically included for EDS study. The outcome of patients was also recorded.

Clinical categorization: All the dengue cases presented in the hospital were categorized clinically in 3 groups: mild, moderate and severe cases following the clinical guideline.

Mild cases of dengue were excluded for the study as they do not have warning signs and symptoms. They were managed in OPD basis, were counseled to go home and advised to take paracetamol, ORS, fruit juice and enough fluid.

Moderate dengue cases were defined as the patients with warning signs and symptoms such as, recurrent vomiting, abdominal pain, tenderness, general weakness, lethargy, restlessness, minor bleeding, pleural effusion, ascites, hepatomegaly, increased hematocrit and the presence of comorbid conditions such as, old age, diabetes, hypertension, pregnancy, coronary artery disease, hemoglobinopathy, 
immunocompromised patient, patient on steroid, anticoagulation or immunosuppressant etc. They were admitted in the ward or cabin for close monitoring and management, as these group of patients may develop severe dengue manifestations at any time. Their management comprised of paracetamol and IV fluid replacement following the algorithm of the guideline. The cases who did not need HDU or ICU care according to the HDU and ICU criteria were not included in this study.

Severe dengue were the patients who had shock, capillary leakage, significant bleeding, severe organ involvement (expanded dengue syndrome) with severe metabolic abnormalities and were required to be admitted or shifted to ICU and HDU. Fluid therapy was immediately initiated, calculating the amount based on body weight and charted on 1-3 hourly basis or even more frequently in the case of shock. The fluid deficit was calculated for correcting dehydration as $5 \%$ deficit plus maintenance using the Holiday-Sagar formula ${ }^{1}$.

Management of severe cases: Patients with severe bleeding, blood transfusion was arranged. However, when blood was not available, shock was managed with proper IV fluid or plasma expander. Patients having thrombocytopenia and active bleeding, the condition was corrected with blood transfusion and/ or platelet transfusion (apheresis). Prophylactic platelet transfusion was considered when platelet level showed $<10,000 / \mathrm{mm} 3$ in absence of bleeding manifestation. Platelet transfusion was also given in patients with prolonged shock with coagulopathy and abnormal coagulation. In case of systemic massive bleeding, platelet transfusion plus red cell transfusion was considered. Use of fresh frozen plasma or cryoprecipitate in coagulopathy with bleeding was given as per the advice ${ }^{1}$. In case of massive hemorrhage, coagulopathy was searched for, tested for PT and APTT specially in patients who had deranged LFT. In presence of prolonged PT, IV vitamin K1 was initiated ${ }^{1}$

Dengue with coinfection and comorbidities: The study excluded the patients with established Chronic Liver Disease (CLD), or Chronic Kidney Disease (CKD) cases, as the study focused only expanded dengue syndrome. However, dengue with diabetes mellitus, hypertension, bronchial asthma, thyroid disease, ischaemic heart disease and stroke (with and without the complication) were admitted according to their severity.

Dengue patients with pregnancy: Pregnant patients with complications of dengue fever were admitted in ICU or HDU. Complications of dengue fever (DF) in pregnant cases depend on the stages of pregnancy like early, late, peripartum and post-partum period. As pregnancy is a hypervolemic condition, fluid replacement were carefully carried out to prevent pulmonary edema and to reduce morbidity and mortality of mother as well as fetus. ${ }^{8}$

\section{Results:}

A total of 343 adult seropositive dengue cases were screened in the study. Of the cases, 187 patients were male $(54.5 \%)$ and 156 patients were female (45.5\%) with male to female ratio $1.2: 1$. The age of all the patients were 18 years and above. Among the patients, $62(18.1 \%)$ cases from July to December 2018 either admitted in ICU or HDU, 45 patients required HDU and 17 patients required ICU supporting care. From January to April 2019, only 3 patients were admitted in the cabin with moderate dengue, not included in the analysis. Total 42 patients had atypical presentations (67.74\%). Of them, 25 (40.3\%) had EDS and 17 (27.4\%) patients had $\mathrm{DSS}^{9}$. The patients who developed EDS characteristically had ascites, plural effusion and edematous gall bladder wall noted in their respective ultra-sonogram. All the patients had high hematocrit, low albumin and rapid fall of platelet count. Most of the patients had hepatitis and electrolyte imbalance.

Dengue with co-infection: Among the patients treated in ICU and HDU, 16 (25.8\%) patients had co-infection, such as Urinary tract infection (12.5\%), Nasolabial herpes (6.25\%), Sepsis with Hospital Acquired Pneumonia (18.75\%), Community Acquired Pneumonia (62.5\%). Among the latter category, 2 (12.5\%) patients developed Acute Respiratory Distress Syndrome (ARDS) and required tracheostomy; while 5 (31.25\%) patients had septic shock, of whom 2 (40\%) patients developed DIC (disseminated intravascular coagulopathy).

Dengue with comorbidities: Of 62 dengue patients, $45(72.58 \%)$ had co-morbidities such as Hypertension (35.55\%), Diabetes Mellitus (31.11\%), Ischaemic Heart Disease (8.88\%), Bronchial Asthma (8.88\%), Pulmonary Hypertension (2.22\%), Acute Myocardial Infarction and Stroke (2.22\%) and Hypothyroidism $(2.22 \%)$. Majority of the comorbid patients $(71.11 \%)$ had atypical presentations due to involvement of other organs (Table 1).

\section{Table-I}

Distribution of patients with notable atypical presentation

\begin{tabular}{llc}
\hline S1 \# & Organs involvement & N (\%) \\
\hline 01 & Myocarditis only & $5(15.6 \%)$ \\
02 & Myocarditis plus other organs & $4(12.5 \%)$ \\
03 & Hepatitis only & $5(15.6 \%)$ \\
04 & Hepatitis plus other organs & $8(25.0 \%)$ \\
05 & Pancreatitis only & $1(3.1 \%)$ \\
06 & Pancreatitis with other organs & $4(12.5 \%)$ \\
07 & Encephalopathy with other organs & $1(3.1 \%)$ \\
08 & Extradural hematoma & $1(3.1 \%)$ \\
09 & Acute renal failure with other organs & $3(9.4 \%)$ \\
\hline & Total & $32(100 \%)$ \\
\hline
\end{tabular}


Dengue with pregnancy: One dengue patient with 33 weeks of pregnancy was admitted at HDU. Two dengue patients were admitted in ICU; one with 13 weeks of pregnancy and another of 34 weeks of pregnancy with Gestational Diabetes Mellitus (GDM). The patient with GDM required emergency LUCS (lower uterine caesarian section) as there was no fetal movement on ultra-sonogram. Her platelet count was low and managed accordingly. The outcome was satisfactory; the baby was alive and was managed in neonatal ICU.

Expanded dengue syndrome: Of 25 cases of EDS, $15(60 \%)$ patients presented with fever and 10 (40\%) cases in the afebrile condition. All of them had malaise, body ache, and headache (12\%). Gastrointestinal symptoms were most common such as, anorexia and nausea (24\%), vomiting (28\%), abdominal pain $(20 \%)$, and $16 \%$ patients with loose motion. As the patients with EDS were in critical condition, so they rushed for immediate care to the emergency department. After resuscitation, all baseline investigations were urgently requested and the laboratory reports were available within an hour. This facilitated to diagnose EDS patients instantly with multi-organ involvement. They were transferred to HDU or ICU as indicated. Further, many patients with EDS were referred to our centre from other hospitals as ICU and HDU facilities are available in this setting. Thus compared to other hospitals, we received more EDS patients.

Virological confirmation: The patients with EDS were virologically confirmed as dengue either by detecting NS1 antigen, dengue RT-PCR or serological test for IgM. Of them, $20(80 \%)$ were positive by NS1, $2(8 \%)$ by RT-PCR and 3 (12\%) were for IgM positive. Necessary biochemical or hematological tests were also carried out and patients were managed accordingly.

Age and sex distribution of EDS patients: Of 25 (7.3\%) EDS patients, 15 (60\%) were male and 10 (40\%) were female with male to female ratio is $3: 2$. The age of admitted patients were 18 yrs and above, and they were arbitrarily stratified into 4 groups (Table 2), such as 18-28, 29-39, 40-50 and 51-60+. The patients of age group 40-50 were mostly affected (52\%) followed by $29-39$ age groups (28\%).

Table-II

Distribution of the patients with EDS by age group ( $n=25)$

\begin{tabular}{lc}
\hline Age group (years) & Number of patients $(\%)$ \\
\hline $18-28$ & $4(8)$ \\
$29-39$ & $7(28)$ \\
$40-50$ & $13(52)$ \\
$51-60+$ & $1(4)$ \\
\hline Total & $25(100)$ \\
\hline
\end{tabular}

- Figure in the parenthesis indicates the percentage $(\%)$ of affected patients.
Blood group in EDS patients: Majority of patients had blood group B positive: 13 (52\%), followed by A positive $6(24 \%)$, O positive $4(16 \%)$ and AB positive 2 (8\%).

Seasonality of EDS: The EDS patients got admitted during the month of July to December 2018 and the monthly pattern of admission was in the figure 1 . Majority of patients developed expanded dengue syndrome in the month of October $8(32 \%)$.

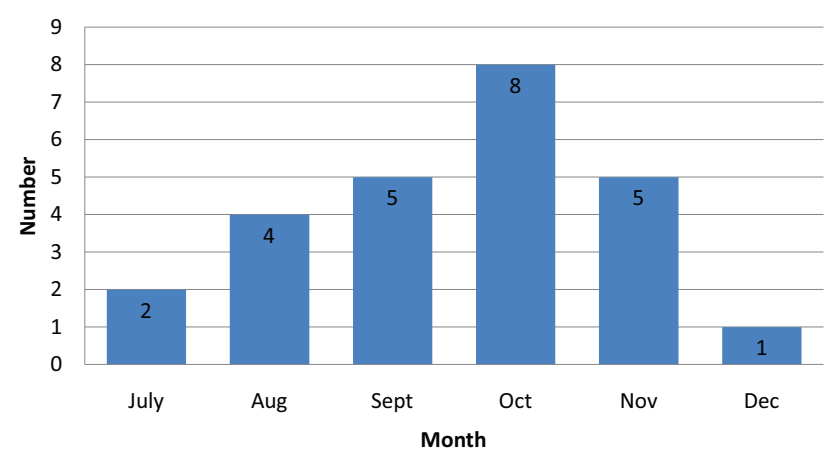

Fig.-1: Numbers of EDS cases by month

EDS DSS patients with comorbidities: Of 32 patients with comorbidities (Table 2), the majority of them showed liver involvement (40.6\%) both singly or together, followed by myocarditis (28.1\%) either singly or with other organs involvement and $15.6 \%$ pancreatitis either singly or with other organs, and 9.4\% acute renal failure, 2 patients had ARDS. One patient had encephalitis, one had compressive myelopathy of spinal cord due to hemorrhage, and 12 patients had associated severe electrolyte imbalance. Myocarditis patients developed bradycardia and had cardiogenic shock, Troponin I was raised. On Echocardiography, they had global hypokinesia and require ionotropic support.

Acute renal failure: Four patients developed acute renal failure who had septicaemic shock and severe electrolyte imbalance. They were managed with dialysis. Acute tubular necrosis may develop in DSS and cause acute kidney injury if fluid therapy is not initiated in time ${ }^{1}$ due to late presentation in hospital. They also had developed rhabdomyolysis.

Mortality associated factors: Four patients who died from the dengue expanded syndrome had septicemia and developed multi-organ dysfunction including acute renal failure. Of 4 patients, 2 patients developed DIC. Their hospital stay extended from was 2 days to 1 month. However, all 4 patients expired in spite of all necessary efforts including dialysis. Among the 4 patients who died, 3 had acute renal failure, their CPK 
was high ranging from $522-16000 \mathrm{U} / \mathrm{L}$. All of them had other organs involvement as well. None of them could survive. Of 25 patients, $68 \%$ had higher values of CRP ( $>10 \mathrm{mg} / \mathrm{L}$ ) with moderately increase (19 to 45 $\mathrm{mg} / \mathrm{L}$ ) in $23.5 \%$, highly increase ( 50 to $>80 \mathrm{mg} / \mathrm{L}$ ) $70.6 \%$ and severely increased $(242 \mathrm{mg} / \mathrm{L})$ in $5.9 \%$. A Taiwan study had similar observation ${ }^{10}$. Serum albumin was done in 10 patients of EDS of which 7 $(70 \%)$ patients had serum albumin $<3 \mathrm{gm} / \mathrm{dl}$.

Overall Management: In addition to symptomatic management of patients with EDS and DSS or with co-infections and co-morbidities, several procedures were performed. Mechanical ventilation required in 17 patients, Tracheostomy in 2, Ionotropic support in 9, Dialysis for 3, Plasma Exchange for 2, Decompressive laminectomy and evacuation of hematoma in 1 , Apheresis in 9, Small unit of platelet for 7, PRBC required for 3, and FFP for 4 patients. In general, hospital stay of dengue patients was variable; 10 patients were released within 5 days, 10 patients between $5-10$ days, 4 patients between $10-15$ days and one patient required to stay for $>20$ days.

\section{Discussion:}

Dengue fever is a mosquito-borne viral disease caused by four closely related and antigenically distinct serotypes of dengue virus, DenV 1-4. Infection with any of 4 serotype confers lifelong homotypic immunity to that particular serotype and provide a brief period (2 yrs) of partial heterotypic immunity to other serotype. However, an individual can eventually be infected by all 4 serotypes. More than one serotype can be in circulation during an outbreak or epidemic. The incidence of dengue have increased dramatically in recent decades, with estimates of $40-50 \%$ of the world's population are at risk for disease in tropical, subtropical and even on temperate areas. The Southeast Asian countries have endemic dengue infection with multiple serotypes of the virus, sometimes develop severe dengue cases. WHO ranked dengue as one of the top ten threats to global health in $2019 .^{2}$

Recent outbreak in Bangladesh in 2019, an overwhelmingly number of dengue cases (over 100,000 cases and $>250$ deaths) was reported, many of them had atypical presentations involving multi-organ failure leading to fatality. This study analyzed data from July 2018 to April 2019 (only 3 cases of 2019 outbreak) However, the content of information in this manuscript nevertheless is very important as it reports from complicated cases clubbed as the EDS who were having atypical presentations of dengue and be useful for the caregivers in managing such cases.
The admitted patients were closely monitored and investigations were carried out for appropriate management and prevention of multi organ failure. The majority of the critical cases were successfully managed. Only 4 patients were discharged on request against medical advice and 4 patients expired (mortality rate of $1.1 \%$ ); which is almost similar to a study in Kerala, India ${ }^{11}$. Among the death cases, DIC was found to be the ultimate cause of death in the Indian study, which is similar to our finding. The outcome of all other patients was uneventful recovery and discharged from the hospital. The hospital stay ranged from 2- 42 days depending on the severity of the disease and other co-morbidities.

In the patients of EDS, mortality rate however, was higher $(16 \%)$, reasons may be multifactorial and can be ascribed to delay in reporting hospital resulting severity and fatality or inappropriate management leading to atypical presentations that caused the development of expanded dengue syndrome, coined by WHO in 2012 that describes cases which do not fall into either dengue haemorrhagic fever (DHF) or dengue shock syndrome (DSS). The atypical manifestations noted in EDS are multisystemic and multifaceted with other organ involvement, such as, liver, brain, heart, kidney and CNS etc. A few cases of EDS have been reported earlier in patients with secondary dengue infection who developed rhabdomyolysis induced acute kidney injury (RAKI) along with intracranial and intraorbital bleeding. ${ }^{12}$ This study presented 25 cases of EDS and 17 cases of DSS with varieties of clinical manifestations associated with comorbidities such as myocarditis, hepatitis, pancreatitis, encephalopathy, extradural hematoma with compressive myelopathy, electrolytes imbalance and acute kidney injury etc. This clinical information is useful and would increase the knowledge sharing and some confidence in caregivers in handling such cases in the upcoming seasons.

Of the laboratory markers, CRP and serum albumin were found to be good predictors of EDS. Higher CRP value and low albumin is a predictor of plasma leakage. Patients of dengue fever usually have an enormous invisible loss of fluid that leads to severe dehydration. A case with severe dehydration, if not treated in time, has multiple consequences including renal failure. A higher value of serum CPK in patients with severe dehydration could be a predictor of impending renal failure. Immediate step to be taken to prevent acute renal failure as the acute necrosis of renal tissue is found to be the cause of $100 \%$ mortality.

The dengue infection is now established as the endemic viral disease in Bangladesh following outbreak ${ }^{13}$ in 
2000; a sero-prevalence study, however, shown its prevalent before that outbreak. The seasonal pattern of the outbreak in 2000 and that of 2019 is similar with predominance of cases occurring in the monsoon and post monsoon period, This is the peak time for favourable transmission when prevailing temperature is in the range of $25^{\circ} \mathrm{C} \pm 5 \mathrm{C}$, relative humidity around $80 \%$ and innumerable small water collection pockets result in high transmission. The number of dengue patients admitted in 2019 so far almost double (1.8 times) the total figure from 2000-2018. The underlying cause of high rise of its incidence also can be attrbuted to disturbed ecology, unplanned urbanization, inadequate source reduction and the scarcity of amphibian species which used to serve as natural mosquito killer during the monsoon. Dhaka was the centre of the outbreak of 2019 but cases were reported from across the country. Risk is present throughout Bangladesh and the year-round with peak transmission during the monsoon season, from June to September or even October. As the case loads increase in the season, the caregivers should be cautious to handle such increase loads of dengue patients each year to avoid the development of complications and/or EDS to reduce the mortality. However, the outbreak or epidemic was known to cycle every 3-5 years.

\section{Conclusion:}

No specific antiviral medication is currently available to treat dengue. The only way to truly prevent dengue virus acquisition is to avoid being bitten by a vector mosquito carrying the dengue virus. This study throws some light on atypical manifestations of dengue, especially during ongoing epidemics. The number of EDS study patients was low, thus a further study using a broad data set is required to define the strategy to prevent the complications of dengue and thereby the deaths. This should address the differentiating markers between DF and DHF so that the onset of critical phase (plasma leakage) could be identified early that will guide the appropriate symptomatic management, careful fluid replacement and to provide required amount of balanced nutrition in order to enhance the early recovery.

\section{Acknowledgement:}

The authors gratefully acknowledge the contributions of the team for their valuable advices and constant encouragement in developing the questionnaire, data collection and organization, suggestions while preparing draft of the manuscript and preliminary and final critical review: The team express sincere thanks to Prof Dr. Syed Atiqul Haq, Prof Dr. Qazi Tariqul Islam, Associate Professor Dr Muhammad Abdur Rahim.

The authors gratefully acknowledge the Square Hospital Authority for allowing the data collection and analysis for this manuscript.
Conflict of interest: The authors have no conflict of interest on this manuscript.

\section{References:}

1. Ashutosh Biswas, Ghan Shyam Pangtey, Akshay Chandra Dhariwal. Indian national guidelines for clinical management of dengue fever article. 2015; volume 113.No 12.Page 196

2. World Health Organization. Global strategy for dengue prevention and control 2012-2020.Geneva; WHO, 2012; Available from: http/bitstream/10665/75303/1/ 978924 1504034-eng.pdf.

3. National guideline for clinical management of Dengue Syndrome. National Malaria Elimination and Aedes Transmitted Disease Control Program .Disease Control Unit. Directorate General of Health Services, Mohakhali, Dhaka-12, 4th edition, 2018: page 4.

4. Rimi Farhana, Khan Fatema Awatef, Hamida Khanum, and Tangin Akter. Prevalence of dengue fevers among the patients of different economic status attended at local hospital in Dhaka. Bangladesh J. Zool, 2014:42(2):161168. https://doi.org/10.3329/bjz.v42i2.23352

5. Quazi Tarikul Islam, Ariful Basher, Robed Amin. Dengue; A practical experience of medical professionals in hospital. J Medicine 2012; 13; 160-166. https:// doi.org/10.3329/jom.v13i2.12751

6. Gubbler DJ. Dengue and dengue hemorrhagic fever. Clin Microbiol Rev. 1998; 11:480 https://doi.org/ 10.1128/CMR.11.3.480

7. Bhatia R, Dash AP, Sunyoto T, Changing epidemiology of dengue in South -East Asia, WHO South -East Asia J Public Health 2013;2:23-7. http://doi.org/10.4103/ 2224-3151.115830. https://doi.org/10.4103/22243151.115830. PMid:28612819

8. Dash AP, Bhatia R, Kalra NL, Dengue in South- East Asia: An appraisal of case management and vector control, Dengue Bulletin.2012; Volume 36..

9. Stuart H.Ralston, Lan D.Penman, Mark W.J. Strachan, Richard P.Hobson. ed. Davidson's principles of Medicine, In Chapter: Acute Medicine and Critical Illness, 23rd ed., Volume -1, page no 190.2018

10. Chien-Chih Chen, Ing-Kit Lee, Jien Wei Liu, Shi-Yu Huang, Lin Wang. Utility of $\mathrm{C}$ reactive protein levels for early prediction of Dengue severity in adults. Kaohsiung Chang Gung Memorial Hospital, Kaohsiung 833, Taiwan.Biomed research international. Research article.Open access. Volume 2015.Article ID 936062.6pages.http//doi. org/10.1155/2015/936062

11. Rachel Daniel, Rajamohanan and Aby Zachariah Philip. A study of clinical profile of dengue fever in Kollam, Kerala, India. dc. date.available 2015-05-01T11:15:46Z. Dengue Bulletin.

12. Smith DS, and Bronze MS. Dengue. In: http:// emedicine.medscape.com/article/215840-print,, may 03,2019: page 1-37

13. Hossain M.A., Mahmuda K, Arjumand F., Nisaluk A and Breiman R.F. Serologic Evidence of Dengue Infection before Onset of Epidemic in 2000, Bangladesh. Emerging Infectious Diseases. 2003 Nov; 9(11): 14111414. https://doi.org/10.3201/eid0911.030117. PMid:14718084 PMCid:PMC3035545. 